\title{
Analysis of microbial community composition and diversity in postoperative intracranial infection using high-throughput sequencing
}

\author{
LIXIN RUAN $^{1}$, DAOWU WU ${ }^{1}$, XINCHONG LI ${ }^{1}$, QICHUAN HUANG ${ }^{1}$, LAIPENG LIN $^{1}$, \\ JIE LIN $^{1}$, LIE CHEN ${ }^{1}$, PEISONG XU ${ }^{2}$, JIE JIN ${ }^{2}$, NINGMIN YANG ${ }^{3}$ and XIANFENG LI ${ }^{1}$
}

${ }^{1}$ Department of Neurosurgery, The People's Hospital of Pingyang, Wenzhou, Zhejiang 325400; Departments of ${ }^{2}$ Research Service and ${ }^{3}$ Clinical Laboratory, Zhiyuan Inspection Medical Institute Co., Ltd., Hangzhou, Zhejiang 310006, P.R. China

Received May 9, 2016; Accepted March 28, 2017

DOI: $10.3892 / \mathrm{mmr} .2017 .7082$

\begin{abstract}
Intracranial infection is one of the most serious complications following neurosurgery. It is well acknowledged that bacteria and fungi are the main pathogens responsible for postoperative intracranial infection. However, the microbial community structure, including composition, abundance and diversity, in postoperative intracranial infection is not fully understood, which greatly compromises our understanding of the necessity and effectiveness of postoperative antibiotic treatment. The present study collected eight cerebrospinal fluid (CSF) samples from patients with intracranial infection following neurosurgical procedures. High-throughput amplicon sequencing for $16 \mathrm{~S}$ rDNA and internal transcribed spacer (ITS) was performed using the Illumina MiSeq platform to investigate the microbial community composition and diversity between treated and untreated patients. Bioinformatics analysis revealed that the microbial composition and diversity in each patient group (that is, with or without antibiotic treatment) was similar; however, the group receiving antibiotic treatment had a comparatively lower species abundance and diversity compared with untreated patients. At the genus level, Acinetobacter and Staphylococcus were widely distributed in CSF samples from patients with postoperative intracranial infection; in particular, Acinetobacter was detected in all CSF samples. In addition, five ITS fungal libraries were constructed, and Candida was detected in three out of four patients not receiving antibiotic treatment, indicating that the fungal infection should be given more attention. In summary, 16S and ITS high-throughput amplicon sequencing were practical methods to identify
\end{abstract}

Correspondence to: Dr Xianfeng Li, Department of Neurosurgery, The People's Hospital of Pingyang, 112 Yahe Road, Wenzhou, Zhejiang 325400, P.R. China

E-mail: pywsjlxf@163.com

Key words: intracranial infection, cerebrospinal fluid, 16S rDNA and ITS amplicon sequencing, microbial community, abundance, diversity pathogens in the different periods of treatment in patients with postoperative intracranial infection. There was a notable difference in microbial composition and diversity between the treated and untreated patients. Alterations in the microbial community structure may provide a signal whether antibiotic treatment worked in postoperative intracranial infection and may assist surgeons to better control the progression of infection.

\section{Introduction}

Postoperative intracranial infection is one of the most serious complications that directly affects the success of craniocerebral operations. Open craniocerebral injury, intracranial lesions, neurosurgery and cerebrospinal fluid leakage due to neurosurgery impair the peripheral tissue of the brain and the blood-brain barrier, significantly increasing the incidence of postoperative infection (1). Despite the administration of preoperative antibiotic prophylaxis, the rate of intracranial infection is $0.8-7 \%$, but would be higher if treatment was not provided (2). Postoperative intracranial infections caused by bacteria, fungi, viruses and parasites have been reported, with symptoms including, headache, lethargy, fever and even death $(3,4)$. Therefore, early diagnosis of postoperative intracranial infection and characterization of infectious pathogens in cerebrospinal fluid (CSF) samples is of great importance.

Currently, bacterial culture of CSF is considered the 'gold standard' in the diagnosis of intracranial infection, guiding the optimal treatment by clinicians. However, bacterial culture is time consuming, usually taking 2-3 days for ordinary bacteria and longer for certain bacterial species such as Mycobacterium tuberculosis to be identified $(5,6)$. Furthermore, bacterial culture from CSF samples is only applicable to a limited number of bacteria, and many factors, including nutrition, culture environment and contamination, influence the positive detection rate (7). For example, Diplococcus pneumoniae, a common bacterium found in CSF, is a fastidious bacterium and its positive rate of bacterial culture is quite low owing to cell death during sample collection and culture (8). Bacterial culture of CSF is not able to meet the requirements of early clinical diagnosis; thus, a new method to identify all of the pathogens in CSF is urgently required. 
Rapidly developing high-throughput sequencing technology has provided an advanced platform for fast detection and determination of bacterial communities in different biological samples $(9,10)$. In prokaryotes, $16 \mathrm{~S}$ rDNA is an important gene that encodes a small ribosomal subunit, and is considered as a basis for species classification owing to its slow rate of evolution over time (11). By sequencing the variable regions with broad-range primers, the bacterial community structure and the degree of evolution in a specific environment may be analyzed (12). Similar to $16 \mathrm{~S}$ rDNA, the internal transcribed spacer (ITS) regions of fungal DNA can also be used as taxonomic markers. Thus, $16 \mathrm{~S}$ rDNA amplicon sequencing (referred to as ' $16 \mathrm{~S}$ sequencing') has been widely applied in human microbiome studies, offering a comprehensive picture of the microbial community in specific samples (13-17). For example, $16 \mathrm{~S}$ sequencing has been used to examine the microbiota of patients with gallstones and diabetes mellitus $(18,19)$. $16 \mathrm{~S}$ sequencing was demonstrated to be able to determine specific pathogens in CSF, including Neisseria meningitidis, Streptococcus pneumoniae and Haemophilus influenzae (20) and, importantly, it was also able to detect microbes that are rarely found or even unknown (21).

In the present study, $16 \mathrm{~S}$ and ITS amplicon sequencing was conducted to determine the microbial composition of CSF samples collected from eight patients with postoperative intracranial infections. Bacterial and fungal community structures (that is, composition, abundance and diversity) were further compared between patient groups with and without antibiotic treatment to confirm similarity and diversity.

\section{Materials and methods}

Patients and samples. CSF samples were collected from eight patients at The Endoscopy Center of The People's Hospital of Pingyang (Zhejiang, China). Seven of the patients were diagnosed with intracranial infection following craniocerebral operations, and one patient (sample 31) has had intracranial fluid leakage for 10 years (see Table I for detailed patient information). Prior to CSF sample collection, four patients (samples 2, 6, 50 and 54) had infections that were controlled with a combination of vancomycin and sulperazone antibiotic treatment, and the other four patients (samples 10, 15, 31 and 53) exhibited high fever and other symptoms, but did not receive antibiotic treatment. CSF samples were collected by lumbar puncture under sterile conditions. For each patient, $1 \mathrm{ml}$ of CSF was collected into a sterile $1.5 \mathrm{ml}$ microcentrifuge tube. The remaining CSF (200-400 $\mu \mathrm{l})$ that was left after routine and biochemical examinations was stored at $-20^{\circ} \mathrm{C}$. All patients (or the families of those patients who were unconscious or slipped into a coma) in this study had been given an oral explanation and signed the informed consent. The present study was carried out in accordance with the protocol approved by Ethics Committee of the Peoples Hospital of Pingyang.

DNA extraction. CSF $(500 \mu \mathrm{l})$ was centrifuged at $12,000 \mathrm{x} \mathrm{g}$ for $1 \mathrm{~min}$ at $4^{\circ} \mathrm{C}$, the supernatant was discarded and the precipitate was used for DNA extraction. To promote the efficient lysis of Gram-positive bacteria and fungi, and to enhance total DNA yields, lysozyme (100 mg/ml; Sigma-Aldrich; Merck KGaA, Darmstadt, Germany) was added to the lysis buffer that was included in the Invitrogen PureLink Genomic DNA Mini kit (Thermo Fisher Scientific, Inc., Waltham, MA, USA). Total DNA was extracted with the Invitrogen PureLink Genomic DNA Mini kit (Thermo Fisher Scientific, Inc.), according to the manufacturer's protocol. The concentration of genomic DNA was quantified with the Qubit dsDNA HS Assay kit (Thermo Fisher Scientific, Inc.). DNA quality and integrity were measured with an Agilent 2100 Bioanalyzer (Agilent Technologies, Inc., Santa Clara, CA, USA) and an Agilent DNA 1000 kit (Agilent Technologies, Inc.), according to the manufacturer's protocol.

Library construction and amplicon sequencing. 16S rDNA and ITS libraries were constructed with a two-round polymerase chain reaction (PCR) amplification protocol; sterile water was used as a negative control. In the first-round PCR, a 470 bp gene segment of the variable (V)3-V4 regions of the bacterial $16 \mathrm{~S}$ rDNA was amplified with a $50 \mu \mathrm{l}$ PCR amplification system, which contained $\sim 50 \mathrm{ng}$ template DNA, $0.2 \mathrm{mM}$ concentration of each PCR primer, $0.2 \mathrm{mM}$ concentration of each deoxynucleoside triphosphate, 2.5 U Platinum Taq DNA polymerase High Fidelity and 1X PCR buffer. All PCR reagents except for primers were obtained from Thermo Fisher Scientific, Inc. The thermocycling conditions consisted of $95^{\circ} \mathrm{C}$ for $3 \mathrm{~min}$, followed by 25 cycles of $95^{\circ} \mathrm{C}$ for $30 \mathrm{sec}$, $60^{\circ} \mathrm{C}$ for $30 \mathrm{sec}$ and $72^{\circ} \mathrm{C}$ for $30 \mathrm{sec}$. Fungal ITS region 1 was amplified with the same PCR amplification system and thermocycling conditions as $16 \mathrm{~S}$ rDNA, except for the primers. The primers used were as follows: $16 \mathrm{~S}$ rDNA, 5'-TCGTCG GCAGCGTCAGATGTGTATAAGAGACAGCCTACGG GNGGCWGCAG-3' (forward; V3) and 5'-GTCTCGTGG GCTCGGAGATGTGTATAAGAGACAGGGACTACHVGG GTWTCTAAT-3' (reverse; V4); ITS region 1, 5'-TCGTCG GCAGCGTCAGATGTGTATAAGAGACAGCTTGGTCAT TTAGAGGAAGTAA-3' (forward) and 5'-GTCTCGTGGGCT CGGAGATGTGTATAAGAGACAGGCTGCGTTCTTCAT

CGATGC-3' (reverse). The PCR products were confirmed by $1.2 \%$ agarose gel electrophoresis. In the second-round PCR, the PCR products from the first-round were purified using Agencourt AMPure XP beads (Beckman Coulter, Inc., Brea, CA, USA), according to the manufacturer's protocol, and used as the template, each sample was attached to a specific barcode and sequencing adapter. Barcodes and sequencing adapters formed part of the primers, and we synthesized the primers based on sequences in a Nextera XT Index kit (cat. no. FC-131-1001; Illumina, Inc., San Diego, CA, USA). Six primers were used, and the sequences of primers were as follows: N701-N704, CAAGCAGAAGACGGCATACGA GAT [i7] GTCTCGTGGGCTCGG; and S501 and S502, AATGATACGGCGACCACCGAGATCTACAC [i5] TCG TCGGCAGCGTC. The i7 and i5 sequences incorporated into the above primers were as follows: N701 i7, TCGCCT TA; N702 i7, CTAGTACG; N703, TTCTGCCT; N704, GCT CAGGA; S501 i5, TAGATCGC; and S502 i5, CTCTCTAT. The PCR conditions were as follows: $95^{\circ} \mathrm{C}$ for $3 \mathrm{~min}$ followed by 12 cycles of $95^{\circ} \mathrm{C}$ for $30 \mathrm{sec}, 65^{\circ} \mathrm{C}$ for $30 \mathrm{sec}$ and $72^{\circ} \mathrm{C}$ for $30 \mathrm{sec}$. The same PCR reagents were used as in first-round PCR. Library concentrations were quantified with an Agilent 2100 Bioanalyzer with a DNA high-sensitivity chip according to the manufacturer's protocol. 16S and ITS high-throughput 
Table I. Clinical information for the eight patients whose cerebrospinal fluids were used for sequencing.

\begin{tabular}{|c|c|c|c|c|c|c|c|c|c|}
\hline $\begin{array}{l}\text { Sample } \\
\text { no. }\end{array}$ & $\begin{array}{c}\text { Age } \\
\text { (years) }\end{array}$ & Sex & Diagnosis & $\begin{array}{l}\text { Antibiotic } \\
\text { treatment }\end{array}$ & $\begin{array}{c}\text { Main } \\
\text { symptoms }\end{array}$ & $\begin{array}{c}\mathrm{Cl} \\
(\mathrm{mM} / \mathrm{l})\end{array}$ & $\begin{array}{c}\text { Glucose } \\
(\mathrm{mM} / \mathrm{l})\end{array}$ & $\begin{array}{c}\text { Hemameba } \\
\left(10^{6} / 1\right)\end{array}$ & $\begin{array}{c}\text { Protein } \\
(\mathrm{g} / \mathrm{l})\end{array}$ \\
\hline 2 & 48 & Male & $\begin{array}{l}\text { Cerebellar } \\
\text { hematoma }\end{array}$ & $\begin{array}{l}\text { Vancomycin, } \\
\text { sulperazone }\end{array}$ & Normothermia & 124.5 & 3.8 & 5 & 1.265 \\
\hline 50 & 53 & Male & $\begin{array}{l}\text { Traumatic } \\
\text { brain injury }\end{array}$ & $\begin{array}{l}\text { Vancomycin, } \\
\text { sulperazone }\end{array}$ & Normothermia & 123.8 & 2.7 & 6,100 & 5.369 \\
\hline 54 & 44 & Male & $\begin{array}{l}\text { Ventricular } \\
\text { hemorrhage }\end{array}$ & $\begin{array}{l}\text { Vancomycin, } \\
\text { sulperazone }\end{array}$ & Normothermia & 113.5 & 1.4 & 200 & 1.836 \\
\hline 6 & 37 & Female & Meningioma & $\begin{array}{l}\text { Vancomycin, } \\
\text { sulperazone }\end{array}$ & Normothermia & 124.1 & 2.3 & 20 & 0.791 \\
\hline 10 & 78 & Female & $\begin{array}{l}\text { Traumatic } \\
\text { brain injury }\end{array}$ & Untreated & $\begin{array}{l}\text { Fever, } \\
\text { headache }\end{array}$ & 92.2 & 0.9 & 510 & 1.568 \\
\hline 15 & 50 & Male & $\begin{array}{l}\text { Cerebellar } \\
\text { hematoma }\end{array}$ & Untreated & $\begin{array}{l}\text { Fever, } \\
\text { headache }\end{array}$ & 128.1 & 5.8 & 1,140 & 1.253 \\
\hline 53 & 75 & Male & $\begin{array}{l}\text { Thalamic } \\
\text { hemorrhage }\end{array}$ & Untreated & $\begin{array}{l}\text { Fever, } \\
\text { headache }\end{array}$ & 113.4 & 3.0 & 950 & 3.152 \\
\hline 31 & 62 & Female & Hypophysoma & Untreated & $\begin{array}{l}\text { Fever, } \\
\text { headache }\end{array}$ & 122.3 & 0.6 & 32,200 & 2.500 \\
\hline
\end{tabular}

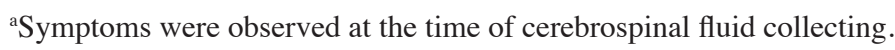

amplicon sequencing was completed by Genewiz (Suzhou, China) using the Illumina MiSeq (Illumina, Inc) sequencing platform to generate $2 \times 250$ bp paired-end reads.

Bioinformatics analysis. Pandaseq (v2.7; http://neufeldserver. uwaterloo.ca/ apmasell/pandaseq_man1.html) was used to merge paired-end reads with a minimumoverlap of $20 \mathrm{bp}$.Primer and barcode sequences were trimmed by Trimmomatic (v0.30; http://www.usadellab.org/cms/index.php?page=trimmomatic) and chimeric sequences were removed with USEARCH (v8.0; http://www.drive5.com/usearch/). The operational taxonomic units (OTU) cluster was performed at the 97\% sequence identity level by using the UCLUST algorithm in the Qiime (v1.7; http:/qiime.org/) bioinformatics software, and a representative OTU was selected from each cluster. Using the Silva_111 16S rRNA database (http://www.arb-silva.de) as a reference, Ribosomal Database Project classifier (http://rdp.cme.msu. edu/classifier/classifier.jsp) was used to assign taxonomic ranks to each OTU. To analyze inter- and intra-population diversity of different samples, $\alpha$ - and $\beta$-diversity of CSF samples was performed. Abundance-based coverage estimators (ACE) and Chao estimators were carried out to calculate community abundance; Shannon and Simpson indices were carried out to evaluate community diversity ( $\alpha$-diversity). $\beta$-diversity analysis was carried out to evaluate the differences in diversity of community composition between the eight CSF samples, which was performed using principal co-ordinates analysis (PCoA) and unweighted pair group method with arithmetic mean (UPGMA) clustering, which were based on distance matrix of unweighted UniFrac.

Statistical analysis. Statistical analyses were performed using SPSS (v19.0; IBM Corp., Armonk, NY, USA). Data are presented as the mean \pm standard deviation. Shannon and Chao 1 indices were compared using the Student's t-test. The Wilcoxon rank-sum test was employed to detect interindividual community differences using $\mathrm{R}$ Project software (https://cran.r-project.org; v3.1.2). $\mathrm{P}<0.05$ was considered to indicate a statistically significant difference.

\section{Results}

Overview of the 16S and ITS sequencing data generated from CSF samples. The present study performed high-throughput sequencing on $16 \mathrm{~S}$ rDNA and ITS, to identify the composition of bacteria and fungus in CSF samples from eight patients with postoperative intracranial infection. 16S rDNA libraries were successfully constructed for all the samples, whereas only 5 ITS libraries were completed (Table II); construction of a negative control library failed. Low-quality reads and chimeric sequences were filtered from the raw data, which left $\sim 1.04$ million reads that met the quality criteria for further bioinformatics analysis. The average length of the $16 \mathrm{~S}$ rDNA and ITS sequences were 465 and $428 \mathrm{bp}$, respectively. The total number of unique OTUs obtained from the $16 \mathrm{~S}$ and ITS sequences were 14,882 and 3,090, respectively, and the number of OTUs identified within each individual sample ranged between 743 and 7147 for 16S, and between 34 and 1621 for ITS. Notably, the samples with the highest number of OTUs were from patients who did not received antibiotic treatment; in particular, the number of OTUs in sample numbers 10,15 and 53 were higher compared with the other samples.

Composition and distribution of microbes in CSF samples. The community structure (comprising composition, abundance and diversity) of bacteria and fungi in the CSF samples are shown in Fig. 1. At the phylum level, there were four bacteria, including Proteobacteria, Firmicutes, Bacteroidetes 
Table II. DNA sequencing data generated from cerebrospinal fluid samples.

\begin{tabular}{|c|c|c|c|c|c|c|c|c|c|c|}
\hline \multirow{2}{*}{$\begin{array}{l}\text { Sample } \\
\text { no. }\end{array}$} & \multicolumn{2}{|c|}{$\begin{array}{l}\text { Raw } \\
\text { data }^{\text {a }}\end{array}$} & \multicolumn{2}{|c|}{$\begin{array}{l}\text { Clean } \\
\text { reads }^{\mathrm{b}}\end{array}$} & \multicolumn{2}{|c|}{$\begin{array}{l}\text { Effective } \\
\text { ratio }^{c}(\%)\end{array}$} & \multicolumn{2}{|c|}{$\begin{array}{c}\text { Average } \\
\text { length (bp) }\end{array}$} & \multicolumn{2}{|c|}{ OTUs } \\
\hline & $16 \mathrm{~S}$ & ITS & $16 \mathrm{~S}$ & ITS & $16 \mathrm{~S}$ & ITS & $16 \mathrm{~S}$ & ITS & $16 \mathrm{~S}$ & ITS \\
\hline 2 & 347,784 & 118,656 & 95,245 & 57,745 & 27.4 & 48.7 & 466.23 & 433.54 & 2,373 & 38 \\
\hline 50 & 126,430 & 0 & 48,445 & 0 & 38.3 & 0 & 462.30 & 0 & 743 & 0 \\
\hline 54 & 108,344 & 0 & 20,276 & 0 & 18.7 & 0 & 473.24 & 0 & 1,216 & 0 \\
\hline 6 & 261,438 & 286,766 & 68,123 & 142,534 & 26.1 & 49.7 & 467.97 & 403.77 & 2,274 & 34 \\
\hline 10 & 176,814 & 342,666 & 75,192 & 69,372 & 42.5 & 20.2 & 461.68 & 450.36 & 5,562 & 1,267 \\
\hline 15 & 338,210 & 352,678 & 144,879 & 82,927 & 33.0 & 23.5 & 461.06 & 436.31 & 7,147 & 1,621 \\
\hline 53 & 261,864 & 251,47 & 111,607 & 53,633 & 42.8 & 21.3 & 461.50 & 415.95 & 6,770 & 885 \\
\hline 31 & 180,686 & 0 & 68,970 & 0 & 32.8 & 0 & 466.55 & 0 & 904 & 0 \\
\hline
\end{tabular}

${ }^{\mathrm{a}}$ Generated with the Illumina MiSeq platform. ${ }^{\mathrm{b}}$ Generated by trimming the primer and special barcode sequences, removing the bases whose quality value was $<20$ on both ends, filtering sequences $<400 \mathrm{bp}$ and removing chimeric sequences. ${ }^{\text {T }}$ The ratio of the clean reads relative to raw reads. ITS, internal transcribed spacer; OTUs, operational taxonomic units.

and Actinobacteria, that were detected at a higher relative abundance than other phyla, with Proteobacteria identified as the most common phylum in all samples (Fig. 1A). For fungi, Ascomycota and Basidiomycota were the major phyla detected in the CSF samples (Fig. 1C). To better characterize the community structure, the microbial and fungal compositions were analyzed at the genus level. The top eight bacterial genera with the highest abundances were Shewanella, Klebsiella, Psychrobacter, Granulicatella, Bifidobacterium, Bacteroides, Staphylococcus and Acinetobacter, which accounted for 10.99-50.44\% of bacterial abundance (Fig. 1B). The top eight fungal genera that were identified included Leptosphaerulina, Cladosporium, Malassezia, Tilletia, Pichia, Saccharomyces, Candida and Peniophora, with a relative abundance of 21.63-76.83\% (Fig. 1D).

Comparison of the community structure in CSF samples. The community structures presented in Fig. 1 were compared between the two groups of patients; that is, those patients who received antibiotic treatment vs. those patients that did not. Bacteroidetes and Firmicutes were the most abundant bacterial phyla detected in patients that did not receive antibiotic treatment (except for patient sample 31), whereas Proteobacteria were more abundant in patients that received treatment. The Wilcoxon rank sum test was performed to detect interindividual community differences. At the genus level, the bacterial composition within each group was similar to each other $(P>0.05)$. No significant differences between the untreated and antibiotic-treated group were observed at the fungal phylum level, however, at genus level, there was no similarity to each other $(\mathrm{P}<0.05)$.

Although some microbes in CSF were highly abundant, the distribution among the samples may be different.Acinetobacter, Staphylococcus, Prevotella and Shewanella were the top four most widely distributed genera, with Acinetobacter being detected in all eight samples (Fig. 2). Cladosporium, Malassezia, Candida and Trichosporon were the top four most widely distributed fungal genera, and Cladosporium was identified in all five samples examined. Notably, Klebsiella was revealed to be highly abundant in untreated patient sample 31, whereas its detection was minimal, if any, in the other seven CSF samples; Staphylococcus was detected in all samples except for sample 31 .

$\alpha$-diversity. To better evaluate the abundance and diversity of pathogens in CSF, $\alpha$-diversity bioinformatics analysis was performed based on OTU data. ACE and Chao estimators were calculated to determine community abundance, and the Shannon and Simpson diversity indices were carried out to evaluate the diversity of community. $\alpha$-diversity results indicated that the postoperative intracranial infections in patients that were treated with antibiotics contained a low abundance microbial community and had reduced within-sample diversity, whereas the untreated patient infections contained high abundance and within-sample diversity (Table III). The $\alpha$-diversity of the group of patients not receiving antibiotic treatment was significantly higher $(\mathrm{P}<0.05)$ compared with the group that did receive antibiotic treatment (Fig. 3).

$\beta$-diversity. $\beta$-diversity analysis was carried out to evaluate the between-sample differences in composition of bacteria of the eight CSF samples (Fig. 4). The results of PCoA indicated that infected patients without antibiotic treatment (samples 10,15 and 53, but not 31) had a similar bacterial and fungal composition (Fig. 4A and B), and a similar situation was observed in the treatment group, with all four samples $(2,6$, 50 and 54) having a similar bacterial composition (Fig. 4A). However, no similarity in the fungal composition between individual samples (samples 2 and 6) in the antibiotic treatment group was observed (Fig. 4B). In addition, the results of heatmap analysis also demonstrated the similarity of the bacterial composition within the antibiotic treatment group and within the untreated group, excluding patient sample 31 (Fig. 4C). However, there was no similarity in the fungal composition within the antibiotic treatment group, but some similarity within the untreated group (Fig. 4D). The present 
A

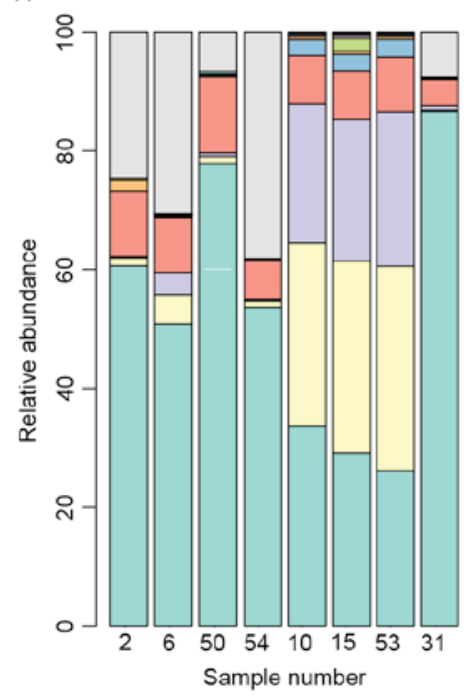

c

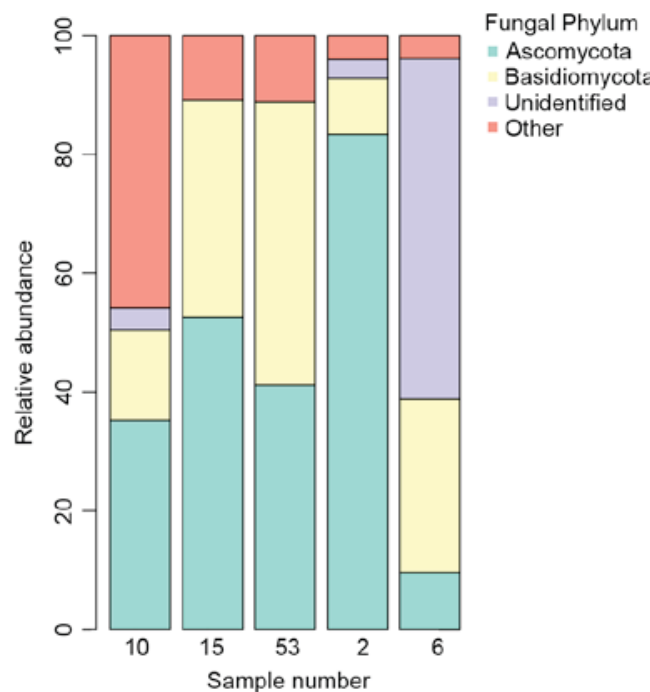

Bacterial Phylum

Proteobacteria*

Firmicutes*

Bacteroidetes ${ }^{*}$

" Actinobacteria*

- Cyanobacteria

Tenericutes

Candidate_division_TM7

- Fusobacteria

Deferribacteres

Acidobacteria

Deinococcus-thermus

Armatimonadetes

Candidate_division_OD1

Planctomycetos

- Euryarchaeota

Gemmatimonadetes

Synergistetes

Spirochaetes

BD1-5

Other
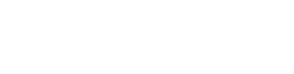

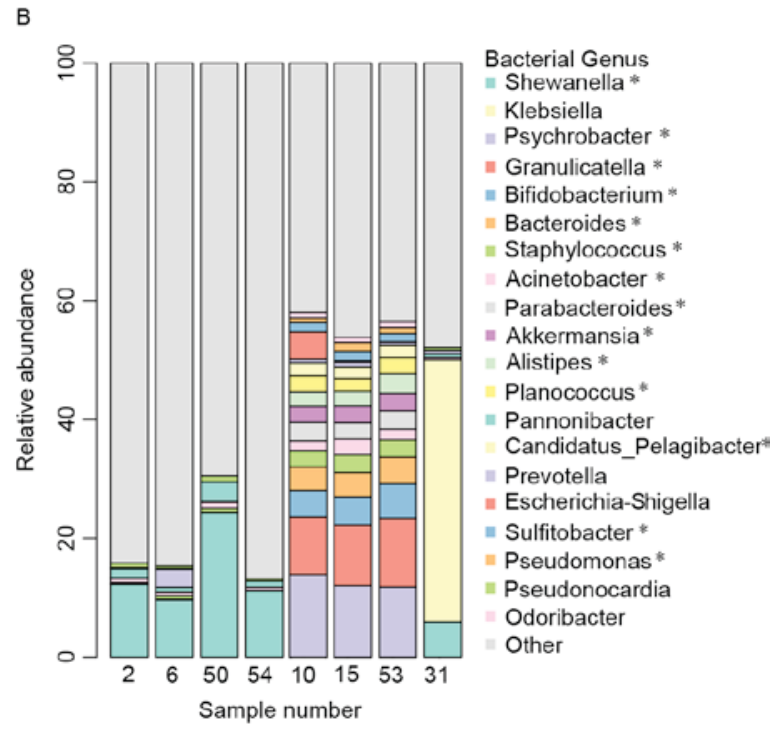

D

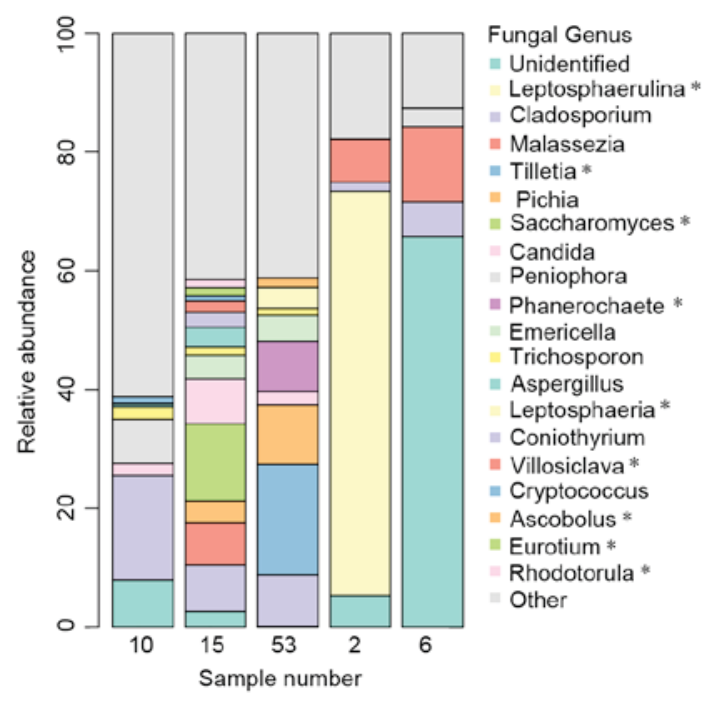

Figure 1. Relative abundance and composition of bacteria and fungi in eight CSF samples. The top 20 bacterial (A) phyla and (B) genera, as well as the top 20 fungal (C) phyla and (D) genera in CSF samples obtained from postoperative patients with intracranial infections treated with antibiotics (samples 2, 6, 50 and 54) or untreated (samples 10,15,31 and 53). 'Other' denotes the relative abundance of bacteria or fungi that were not in the top 20. CSF, cerebrospinal fluid. ${ }^{*} \mathrm{P}<0.05$, untreated vs. antibiotic treatment group.
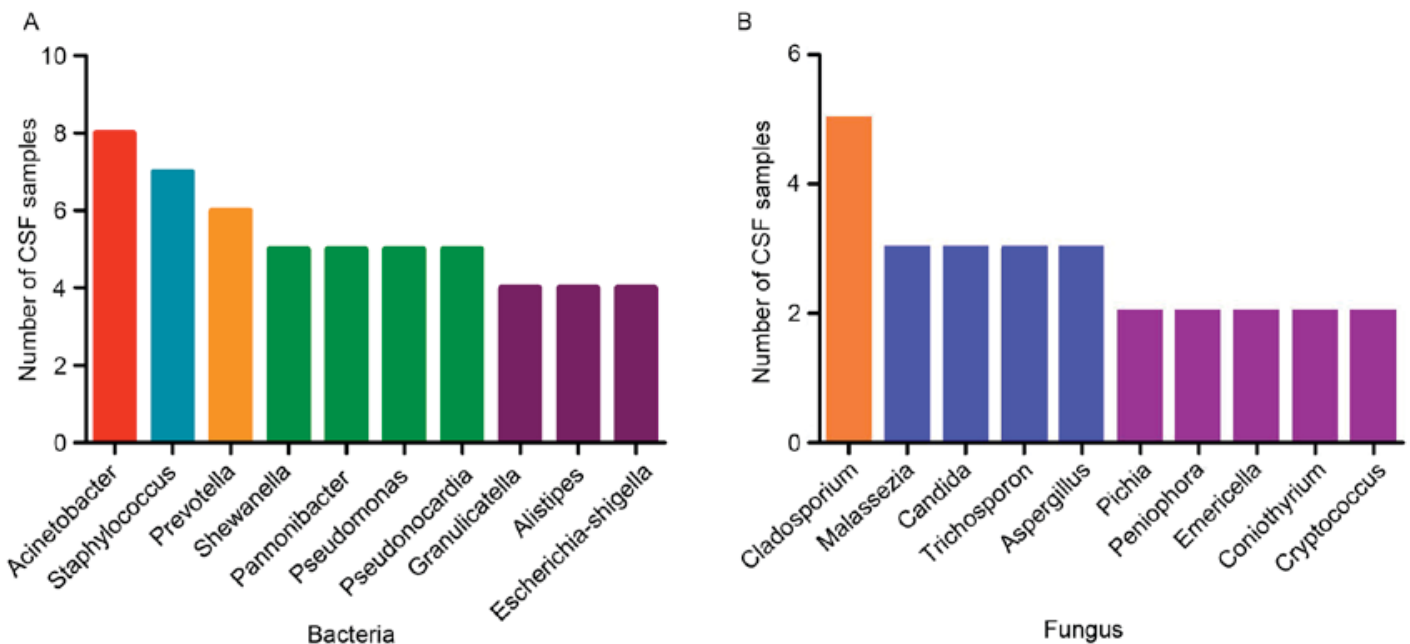

Figure 2. Distribution of pathogens in eight CSF samples. (A) Distribution of bacteria at the genus level in eight CSF samples. (B) Distribution of fungi at the genus level in five of the eight CSF samples. CSF, cerebrospinal fluid. 
Table III. Indices of bacterial alpha diversity in cerebrospinal fluid samples.

\begin{tabular}{|c|c|c|c|c|c|c|c|c|}
\hline \multirow[b]{2}{*}{ Sample no. } & \multicolumn{2}{|c|}{$\mathrm{ACE}^{\mathrm{a}}$} & \multicolumn{2}{|c|}{$\mathrm{Chao}^{\mathrm{a}}$} & \multicolumn{2}{|c|}{ Shannon $^{\mathrm{b}}$} & \multicolumn{2}{|c|}{ Simpson $^{\mathrm{b}}$} \\
\hline & $16 \mathrm{~S}$ & ITS & $16 \mathrm{~S}$ & ITS & $16 \mathrm{~S}$ & ITS & $16 \mathrm{~S}$ & ITS \\
\hline 2 & 2,697 & 38 & 2,537 & 38 & 5.56 & 1.88 & 0.90 & 0.52 \\
\hline 50 & 860 & 0 & 799 & 0 & 3.93 & 0 & 0.84 & 0 \\
\hline 54 & 1,631 & 0 & 1,497 & 0 & 5.92 & 0 & 0.91 & 0 \\
\hline 6 & 2,618 & 34 & 2,463 & 34 & 6.65 & 2.54 & 0.94 & 0.65 \\
\hline 10 & 7,684 & 1,387 & 7,307 & 1,321 & 8.28 & 4.91 & 0.98 & 0.93 \\
\hline 15 & 8,665 & 1,776 & 8,203 & 1,691 & 8.51 & 6.24 & 0.98 & 0.96 \\
\hline 53 & 8,826 & 926 & 8,429 & 896 & 8.39 & 5.23 & 0.98 & 0.95 \\
\hline 31 & 1,068 & 0 & 998 & 0 & 4.13 & 0 & 0.79 & 0 \\
\hline
\end{tabular}

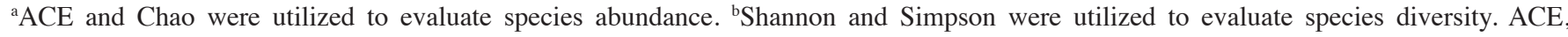
Abundance-based coverage estimators.

A

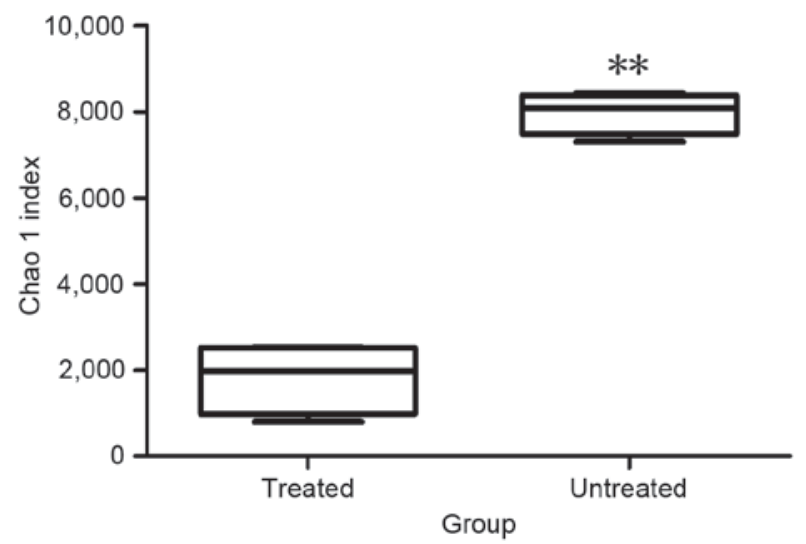

B

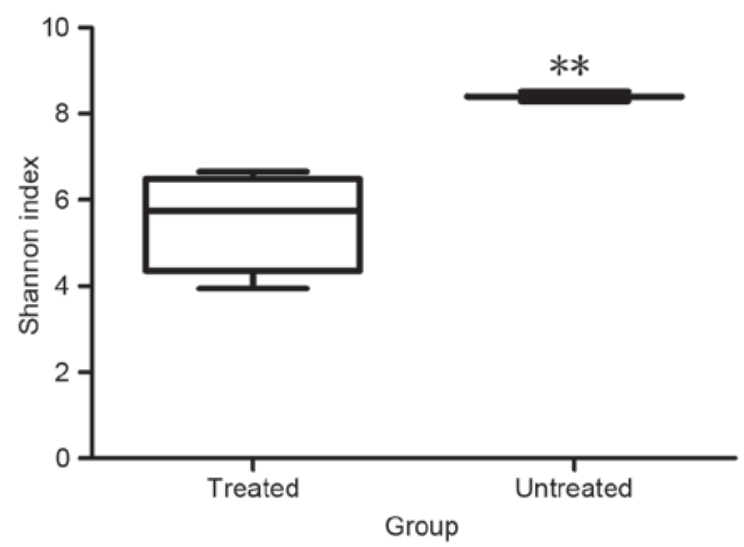

Figure 3. Bacterial $\alpha$-diversity in cerebrospinal fluid samples from eight patients with postoperative intracranial infection that have received antibiotic treatment or were untreated. (A) Chao index for bacterial $\alpha$-diversity. (B) Shannon index for bacterial $\alpha$-diversity. $n=4$ per group. ${ }^{* *} \mathrm{P}<0.01$ vs. antibiotic treatment group.

study performed UPGMA analysis based on distance matrix of unweighted UniFrac to cluster CSF samples, and two clusters were clearly generated. One cluster was composed of the four samples from bacterial-infected patients who had been treated with antibiotics, as well as the untreated patient sample 31 , and the other cluster was composed of the remaining samples from bacterial-infected patients (samples 10, 15 and 53) without antibiotic treatment (Fig. 4E). Simultaneously, the untreated and antibiotic treatment groups were clustered into two separate groups in fungal-infected patients (Fig. 4F). $\beta$-diversity analysis also indicated a notable difference in the CSF samples between patients with infections that had been treated with antibiotics and those without antibiotic treatment.

\section{Discussion}

Intracranial infection is a common complication following neurosurgery, with an incidence rate of postoperative infection at $\sim 10 \%$. Previous studies have reported that postoperative infections may be caused by CSF leakage, drainage, surgery duration, longer hospital stays and multiple craniotomies (22-25). Although a number of previous studies have focused on bacterial infection and have identified Gram-positive Staphylococcus aureus as a main infection agent of CSF, fungal infections are also an important risk that should not be ignored $(26,27)$. To characterize the pathogens of intracranial infection, bacterial culture has been a gold standard for CSF samples. However, owing to the specificity of bacterial cultures, many potential pathogens could not be cultured and identified. However, high-throughput amplicon sequencing has provided an improved picture of the bacterial composition, as well as the fungal composition, in human CSF samples (28). To investigate microbial community structure, the present study performed $16 \mathrm{~S}$ rDNA sequencing for bacteria and ITS sequencing for fungus on eight CSF samples from patients with postoperative infections following neurosurgery.

Candida, Aspergillus and Cryptococcus were the main fungal pathogens to be identified by previous studies $(26,27)$; however, many previous studies have focused on bacterial infection and 
A

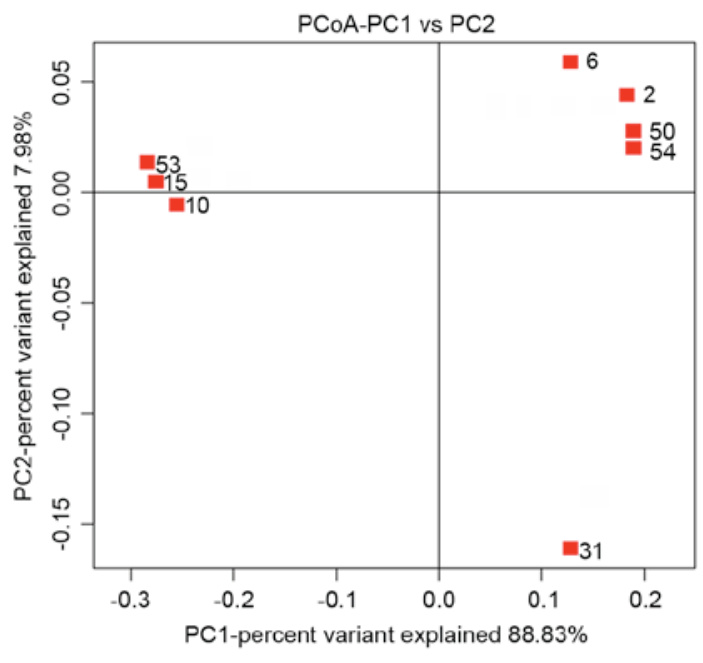

c

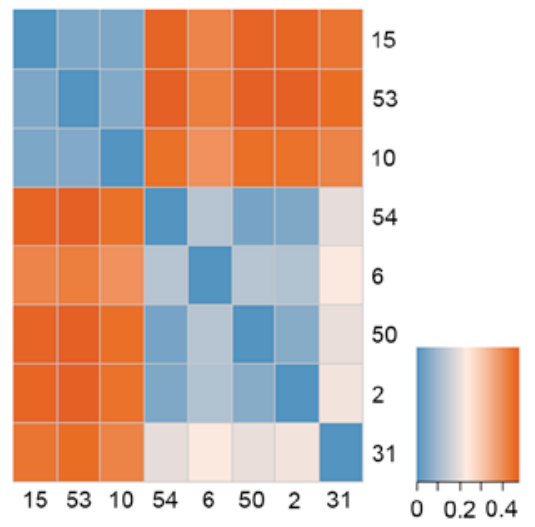

$E$

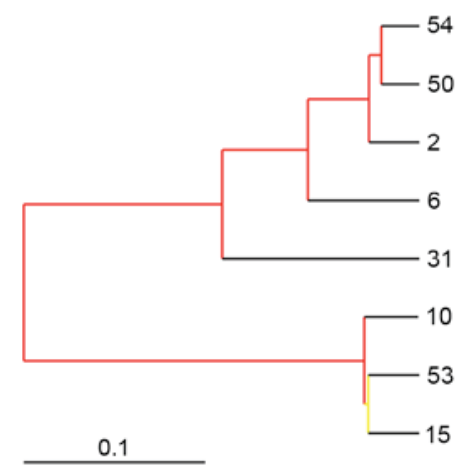

B

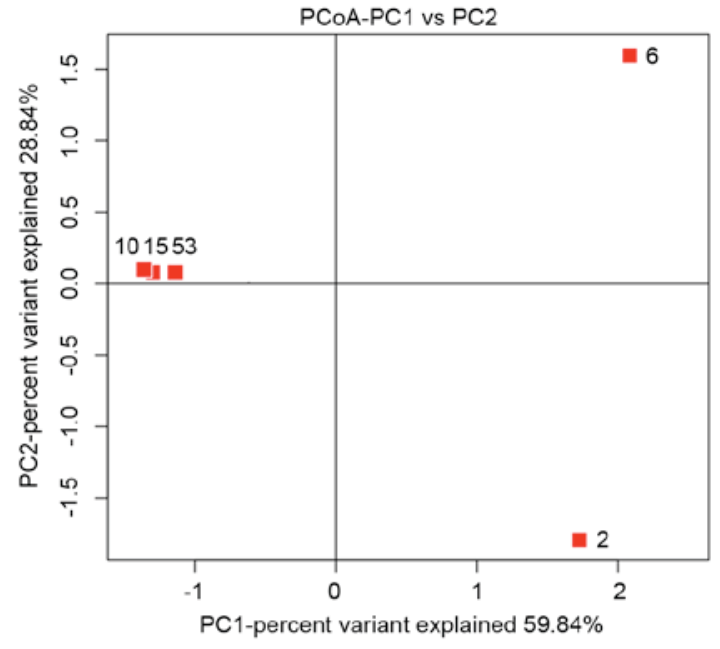

D

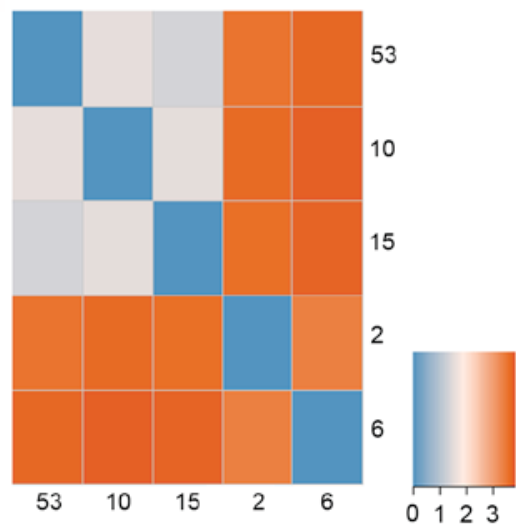

F

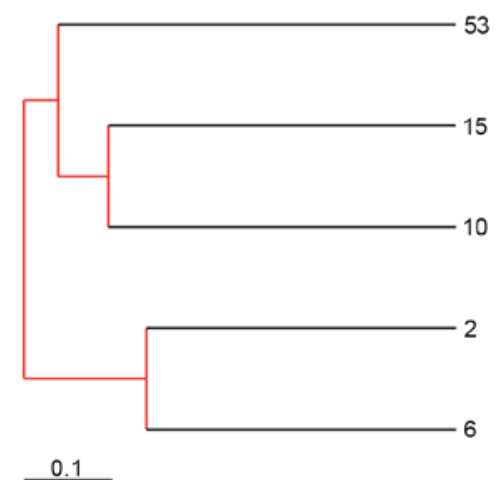

Figure 4. Microbial $\beta$-diversity in CSF samples from patients with postoperative intracranial infection with or without antibiotic treatment. The PCoA plots of (A) bacterial and (B) fungal community in the CSF samples. The heatmap of unweighted UniFrac distance for (C) bacterial and (D) fungal community in the CSF samples. Unweighted pair group method with arithmetic mean cluster results for (E) bacterial and (F) fungal community in the CSF samples. $\mathrm{n}=8$ for bacteria; $\mathrm{n}=5$ for fungus. CSF, cerebrospinal fluid; PCoA, Principal co-ordinates analysis.

ignored fungal infection, indicating that fungal infections should be given more attention. In the present study, CSF samples were examined by $16 \mathrm{~S}$ and ITS sequencing, which involved the successful construction of $16 \mathrm{~S}$ rDNA libraries for all eight of the samples, and an ITS library was completed for five of the CSF samples (including two from patients treated with antibiotics and three from untreated patients). The present results demonstrated that bacterial infections were more frequent than fungal infections in CSF samples. Therefore, it was speculated that although fungal infection can be detected, bacterial infections are predominant in postoperative intracranial infection. Of note, the ITS data revealed that Candida infection was present in the three CSF samples examined from untreated patients, whereas it was not detected in the two samples examined from patients with antibiotic treatment. As the role of pathogens in the development of postoperative 
intracranial infection is complex, and various pathogens may contribute to any individual case, it is important to identify the composition of pathogens in each individual to allow the rational use of antibiotics. Results from the present study confirmed that ITS sequencing was an efficient method to identify fungal infection and that Candida may be a major risk factor of postoperative intracranial infection, similar to a previous report (29). Although Cladosporium also had a higher abundance in untreated patients, no similar reports were identified in the literature. At the genus level, bacterial infections within each group appeared to be similar to each other on the distribution of biological community, except sample 31 in the untreated group as it clearly differed compared with the other untreated samples (10, 15 and 53), whereas fungal infections were more varied within each group.

At the phylum level, Proteobacteria, Firmicutes, Bacteroidetes and Actinobacteria were identified as the most prevalent bacterial infections, and this finding was consistent with a previous report (28). At genus level, Acinetobacter, Staphylococcus and Prevotella were top three genera in the distribution of bacterial infection, with Acinetobacter detected in all CSF samples. Acinetobacter baumannii are Gram-negative bacteria with a high frequency of detection in postoperative intracranial infections (24). Staphylococcus is widely distributed on human skin, which is an important source of hospital cross infection and is a main risk factor for postoperative intracranial infection $(24,30)$. In agreement with a previous report (20), results from the present study demonstrated that Acinetobacter and Staphylococcus were the most prevalent bacteria in postoperative intracranial infection. Comparison of the composition and distribution in the two groups of patients, with or without antibiotic treatment, revealed that Bacteroidetes and Firmicutes were more abundant in patients without antibiotic treatment (except for patient sample 31), whereas Proteobacteria were more abundant in patients receiving treatment. In addition, Staphylococcus and Acinetobacter, which are commonly associated with intracranial bacterial infection, were higher in the untreated group compared with the treated group (except for patient sample 31), indicating that antibiotic treatment could significantly eliminate these main pathogens. Although other types of bacteria had a higher abundance compared with Staphylococcus and Acinetobacter in the untreated group and were also increased compared with the treated group, few studies have reported infections with these bacterial groups to be associated with intracranial bacterial infection.

The results of bioinformatics analysis revealed that the OTUs and $\alpha$-diversity indices of the untreated patient group were significantly higher than in patients receiving antibiotic treatment, indicating that species abundance and diversity were more plentiful. This result may be due to antibiotic treatment eliminating various types of bacteria and fungi, which results in reduced species diversity. Alterations in the microbial community structure may provide a signal as to whether antibiotic treatment worked in patients with postoperative intracranial infection and may assist surgeons to better control the progression of infection.

The present study also demonstrated that the community structure of untreated patient CSF sample 31 was notably different from others: Species abundance and microbial diversity were dominated by Klebsiella. Klebsiella are conditional pathogenic bacteria that are extensively distributed on the mucosal surfaces of humans, and are known to infect people with immune deficiency syndromes such as diabetes mellitus (31). Examination of the medical history revealed that the patient from whom CSF sample 31 was obtained was suffering from diabetes and CSF leakage, which may be related to the type of infection observed in this patient. In cases of long-term infection, a normal community structure similar to that of a healthy adult may be destroyed; thus, Klebsiella may develop into a dominant bacterial taxon and inhibit the growth of other bacteria. Although this was the only sample collected from a patient with CSF leakage, it serves as a reminder that bacterial distribution may be effected by additional factors in postoperative intracranial infection; particularly, if the patient is suffering from other diseases simultaneously.

The present study does have some limitations such as the sample size, which was the biggest problem in this study. Another problem is that multiple samples could not be obtained from the same patient at different time points, because the patient may not be able to bear the lumbar puncture procedure more than once. Additional CSF samples from patients with postoperative intracranial infections in different periods of treatment are being collected to validate the present results and conclusions, with attempts at collecting specimen from the same patient whenever possible.

In conclusion, $16 \mathrm{~S}$ and ITS amplicon sequencing were demonstrated to be practical methods for identifying pathogens in the development of intracranial infection. The present study revealed that infections that are being controlled with antibiotic treatment exhibit reduced within-sample diversity compared with untreated infections. The results provided proof that an alteration of community structure may be considered as a signal of the efficacy of antibiotic treatment in patients with postoperative intracranial infection. In addition, future studies that investigate changes in the microbial CSF of patients with postoperative intracranial infection following different durations of antibiotic treatment and different lengths of time after antibiotic treatment are required.

\section{Acknowledgements}

The present study was financially supported by the Public Technology Application Research of Zhejiang Province Science and Technology Hall (grant nos. 2017C33177 and 2016C33242) and the Medical and Health Science and Technology Plan Project of Zhejiang Province (grant no. 2015KYB371). We also gratefully acknowledge Miss Liyun Zheng (Hangzhou Zhiyuan Medical Inspection Insititute, Hangzhou, China) for assistance with Photoshop, and Mrs. Hui Bai (Beijing Institute of Radiation Medicine, Beijing, China) for proofreading of this manuscript.

\section{References}

1. Liu CX, Wan DJ, Li JL, Yang H and Chao GQ: Clinical analysis on intracranial infection after craniocerebral operation. Chinese J Nosocomiol 10: 4-7, 2000.

2. Dashti SR, Baharvahdat H, Spetzler RF, Sauvageau E, Chang SW, Stiefel MF, Park MS and Bambakidis NC: Operative intracranial infection following craniotomy. Neurosurg Focus 24: E10, 2008. 
3. Smuts H, Botha G, Stanley A and Hardie D: Viruses in CSF of HIV patients with acute and late onset neurological conditions detected by NGS. International J Infectious Diseases 21: 124, 2014.

4. Maia CS, Monteiro MC, Gavioli EC, Oliveira FR, Oliveira GB and Romão PR: Neurological disease in human and canine leishmaniasis-clinical features and immunopathogenesis. Parasite Immunol 37: 385-393, 2015.

5. Wu HM, Cordeiro SM, Harcourt BH, Carvalho M, Azevedo J, Oliveira TQ, Leite MC, Salgado K, Reis MG, Plikaytis BD, et al: Accuracy of real-time PCR, Gram stain and culture for Streptococcus pneumoniae, Neisseria meningitidis and Haemophilus influenzae meningitis diagnosis. BMC Infect Dis 13: 26, 2013

6. Shen J, Guan Y, Zhang J, Tang J, Lu X and Zhang C: Application of microarray technology for the detection of intracranial bacterial infection. Exp Ther Med 7: 496-500, 2014.

7. Relman DA, Schmidt TM, MacDermott RP and Falkow S: Identification of the uncultured bacillus of Whipple's disease. N Engl J Med 327: 293-301, 1992.

8. Wang Y, Wang CQ and Wang XH: Etiology and Antimicrobial Susceptiblity of Children with Bacterial Meningitis. J Appl Clin Pediatr 21: 355-356, 2006.

9. Han $\mathrm{H}, \mathrm{Hu} \mathrm{ZY}$ and Wu BY: Research advancement on methods of gene diagnosis of bacteriology in cerebrospinal fluid. Med Recapitulate 16: 2855-2857, 2010.

10. Jaton K, Sahli R and Bille J: Devlopment of polymerase chain reaction assay for detection of Listeria monocytogenes in clinical cerebrospinal fluid samples. J Clin Microbiol 30: 1931-1936, 1992.

11. Woese CR: Bacterial evolution. Microbiol Rev 51: 221-271, 1987.

12. Salipante SJ, Sengupta DJ, Rosenthal C, Costa G, Spangler J, Sims EH, Jacobs MA, Miller SI, Hoogestraat DR, Cookson BT, et al: Rapid 16S rRNA next-generation sequencing of polymicrobial clinical samples for diagnosis of complex bacterial infections. PLoS One 8: e65226, 2013.

13. Zhou X, Ren L, Meng Q, Li Y, Yu Y and Yu J: The next-generation sequencing technology and application. Protein Cell 1: 520-536, 2010.

14. Thomas RK, Nickerson E, Simons JF, Jänne PA, Tengs T, Yuza Y, Garraway LA, LaFramboise T, Lee JC, Shah K, et al: Sensitive mutation detection in heterogeneous cancer specimens by massively parallel picoliter reactor sequencing. Nat Med 12 : $852-855,2006$

15. Wang J, Wang W, Li R, Li Y, Tian G, Goodman L, Fan W, Zhang J, Li J, Zhang J, et al: The diploid genome sequence of an Asian individual. Nature 456: 60-65, 2008 .

16. Shen Y, Sarin S, Liu Y, Hobert O and Pe'er I: Comparing platforms for C. Elegans mutant identification using high-throughput whole-genome sequencing. PLoS One 3: e4012, 2008.

17. Gabor E, Liebeton K, Niehaus F, Eck J and Lorenz P: Updating the metagenomics toolbox. Biotechnol J 2: 201-206, 2007.
18. Shen H, Ye F, Xie L, Yang J, Li Z, Xu P, Meng F, Li L, Chen Y, Bo X, et al: Metagenomic sequencing of bile from gallstone patients to identify different microbial community patterns and novel biliary bacteria. Sci Rep 5: 17450, 2015.

19. Qin J, Li Y, Cai Z, Li S, Zhu J, Zhang F, Liang S, Zhang W, Guan Y, Shen D, et al: A metagenome-wide association study of gut microbiota in type 2 diabetes. Nature 490: 55-60, 2012.

20. Cui X, Yu H, Wang Y, Li S and Liu L: Distribution and antimicrobial resistance analysis of pathogens in CSF of postoperative CNS infection. Chinese J Pharmacoepidemiology 24: 33-37, 2015.

21. Ward DM, Weller R and Bateson MM: 16S rRNA sequences reveal numerous uncultured microorganisms in a natural community. Nature 345: 63-65, 1990.

22. Kourbeti IS, Jacobs AV, Koslow M, Karabetsos D and Holzman RS: Risk factors associated with postcraniotomy meningitis. Neurosurgery 60: 317-326, 2007.

23. Reichert MC, Medeiros EA and Ferraz FA: Hospital-acquired meningitis in patients undergoing craniotomy: Incidence, evolution, and risk factors. Am J Infect Control 30: 158-164, 2002

24. Zhan R, Zhu Y, Shen Y, Shen J, Tong Y, Yu H and Wen L: Post-operative central nervous system infections after cranial surgery in China: Incidence, causative agents, and risk factors in 1,470 patients. Eur J Clin Microbiol Infect Dis 33: 861-866, 2014.

25. Lin C, Zhao X and Sun H: Analysis on the risk factors of intracranial infection secondary to traumatic brain injury. Chin J Traumatol 18: 81-83, 2015.

26. Kim JP, Park BJ, Lee MS and Lim YJ: Occlusion of the Internal Carotid Artery due to Intracranial Fungal Infection. J Korean Neurosurg Soc 49: 186-189, 2011.

27. Alonso R, Pisa D, Marina AI, Morato E, Rábano A, Rodal I and Carrasco L: Evidence for fungal infection in cerebrospinal fluid and brain tissue from patients with amyotrophic lateral sclerosis. Int J Biol Sci 11: 546-558, 2015.

28. Liu A, Wang C, Liang Z, Zhou ZW, Wang L, Ma Q, Wang G, Zhou SF and Wang Z: High-throughput sequencing of $16 \mathrm{~S}$ rDNA amplicons characterizes bacterial composition in cerebrospinal fluid samples from patients with purulent meningitis. Drug Des Devel Ther 9: 4417-4429, 2015,

29. Geers TA and Gordon SM: Clinical significance of candida species isolated from cerebrospinal fluid following neurosurgery. Clin Infect Dis 28: 1139-1147, 1999.

30. Tattevin P, Schwartz BS, Graber CJ, Volinski J, Bhukhen A, Bhukhen A, Mai TT, Vo NH, Dang DN,Phan TH, et al: Concurrent epidemics of skin and soft tissue infection and bloodstream infection due to community-associated methicillin-resistant Staphylococcus aureus. Clin Infect Dis 55: 781-788, 2012.

31. Podschun R and Ullmann U: Klebsiella spp. as nosocomial pathogens: Epidemiology, taxonomy, typing methods, and pathogenicity factors. Clin Microbiol Rev 11: 589-603, 1998. 